Pure and Applied Mathematics Quarterly

Volume 3, Number 3

(Special Issue: In honor of

Leon Simon, Part 1 of 2$)$

$785-800,2007$

\title{
Interior gradient estimates and existence theorems for constant mean curvature graphs in $M^{n} \times R$
}

\author{
Joel Spruck
}

Dedicated to Leon Simon on the occasion of his 60th birthday

\section{INTRODUCTION}

In this paper we establish a priori interior gradient estimates and existence theorems for n dimensional graphs $S=\{(x, u(x)): x \in \Omega\}$ of constant mean curvature $H>0$ in an $n+1$ dimensional Riemannian manifolds of the form $M^{n} \times R$ where $M^{n}$ is simply connected and complete and $\Omega$ is a bounded domain in M. Our aim is to illustrate the use of intrinsic methods that hold in great generality to obtain apriori estimates. In particular, we shall solve the Dirichlet problem for constant mean curvature graphs analogous to the results of Serrin [14, 15].

If $d s^{2}=\sigma_{i j} d x_{i} d x_{j}$ is a local Riemannian metric on $\mathrm{M}$, then $M \times R$ is given the product metric $d s^{2}+d t^{2}$ where $\mathrm{t}$ is a coordinate for $R$. As we shall see in the next section, the height function $u(x) \in C^{2}(\Omega)$ satisfies the divergence form equation

$$
\operatorname{div} \frac{D u}{W}=n H(x), W=\left(1+|D u|^{2}\right)^{1 / 2}
$$

Received January 16, 2006.

Research supported in part by NSF grant DMS0306197 . 
where the divergence and gradient $D u$ are taken with respect to the metric on M. Equivalently, equation (1.1) can be written in non-divergence form

$$
\frac{1}{W} g^{i j} D_{i} D_{j} u=n H(x),
$$

where $D$ denotes covariant differentiation on $\mathrm{M}$ and

$$
g^{i j}=\sigma^{i j}-\frac{u^{i} u^{j}}{W^{2}}, u^{i}=\sigma^{i j} D_{j} u
$$

We can now state the main results of this paper. Let $\mathrm{P}$ be a point in $\Omega$ and suppose that the geodesic ball $B_{\rho}(P) \subset \Omega$ for $\rho<R(P)$, the injectivity radius of $\mathrm{M}$ at $\mathrm{P}$.

Theorem 1.1.. Let $u \in C^{3}(\Omega)$ be a non-negative solution of (1.1). Then

$$
\left.|W(P)| \leq 32 \max \left(1,\left(\frac{u(P)}{\rho}\right)^{2}\right)\right) e^{16 C u(P)} e^{16 C\left(\frac{u(P)}{\rho}\right)^{2}}
$$

for a constant $C$ independent of $u$, but depending on the $C^{1}$ norm of $H(x)$, a lower bound for the sectional curvatures of $M$ and an upper bound for $\Delta d^{2}$ on $\Omega$

An interior gradient estimate for any bounded solution of (1.1) follows inmediately from Theorem 1.1.

As an application of Theorem 1.1 we have the following non-constructive apriori lower height estimate for constant mean curvature graphs.

Theorem 1.2.. Let $\Omega$ be a bounded domain with $C^{2}$ boundary whose inward mean curvature satisfies the condition $\mathcal{H} \geq \frac{n}{n-1} H+\epsilon$ for some $\epsilon>0$. Let $u$ be a solution of (1.1) for $H(x) \equiv H>0$ constant in $\Omega$ and suppose $u \geq 0$ on $\partial \Omega$. Then there exists $C>0$ independent of $u$ such that $u>-C$ in $\Omega$.

The example $H=\frac{n-1}{n}, \Omega=B_{R} \subset H^{n}$ with $R$ large shows that we may not take $\epsilon=0$.

It is also of interest to consider graphs moving by mean curvature. Let $\Omega_{T}=$ $\Omega \times(0,2 T)$; then $u(x, t)$ satisfies

$$
u_{t}=g^{i j} D_{i} D_{j} u
$$


Theorem 1.3.. Let $u(x, t) \in C^{3}\left(\Omega_{T}\right) \cup C^{0}\left(\overline{\Omega_{T}}\right)$ be a non-negative solution of (1.4). Then,

$$
W(P, T) \leq 32 \max \left(1, \frac{u(P)^{2}}{\rho}\right) e^{16 C\left(u(P)+\frac{u(P)^{2}}{\rho^{2}}+\frac{u(P)^{2}}{T}\right)}
$$

with $C$ independent of $u$, but depending on a lower bound for the Ricci curvature of $M$ and an upper bound for $\Delta d^{2}$ on $\Omega$.

We next turn to existence theorems. For simplicity we consider only constant mean curvature graphs but analogues of Serrin's results for prescribed mean curvature also hold.

Theorem 1.4.. Let $\Omega$ be a bounded domain with $C^{2}$ boundary whose inward mean curvature satisfies the condition $\mathcal{H} \geq \frac{n}{n-1} H>0$. Suppose in addition that the Ricci curvature of $M$ satisfies $\operatorname{Ric}(M) \geq-\frac{n^{2}}{n-1} H^{2}$ on $\Omega$. Then the Dirichlet problem

$$
\begin{aligned}
\operatorname{div} \frac{D u}{W} & =n H \text { in } \Omega \\
u & =\phi \text { on } \partial \Omega
\end{aligned}
$$

is uniquely solvable for arbitrary continuous boundary data $\phi$.

The condition on $\operatorname{Ric}(M)$ insures that the mean curvature of the inward parallel surfaces is increasing as a function of distance. This implies $-\Delta d \geq n H$ which is used in a barrier argument. There is further discussion of other possible assumptions (that give sharp results) in Section 5. Using Theorem 1.3 we may dispense altogether with the condition on Ricci curvature.

Theorem 1.5.. Let $\Omega$ be a bounded domain with $C^{2}$ boundary whose inward mean curvature satisfies the condition $\mathcal{H} \geq \frac{n}{n-1} H+\epsilon$ for some $\epsilon>0$. Then the Dirichlet problem

$$
\begin{aligned}
\operatorname{div} \frac{D u}{W} & =n H \text { in } \Omega \\
u & =\phi \text { on } \partial \Omega
\end{aligned}
$$


is uniquely solvable for arbitrary continuous boundary data $\phi$.

As is well-known from the work of Finn and Serrin, our existence theorems cannot in general be improved except for constant boundary data in certain cases. An example of such result (which is well known for $M=R^{n}$ or $M=S^{n}$ ) is the following.

Theorem 1.6.. Let $\Omega$ be a bounded domain with $C^{2}$ boundary whose inward mean curvature satisfies the condition $\mathcal{H} \geq(1+\epsilon) H$ for some $\epsilon>0$ and assume that that the Ricci curvature of $M$ is non-negative on $\Omega$. Then the Dirichlet problem

$$
\begin{aligned}
\operatorname{div} \frac{D u}{W} & =n H \text { in } \Omega \\
u & =0 \text { on } \partial \Omega
\end{aligned}
$$

is uniquely solvable.

For mean curvature flow, we have the corresponding existence result as in the Euclidean case (see Huisken [7] and Oliker-Uraltseva [11, 12]).

Theorem 1.7.. Let $\Omega$ be a bounded domain with $C^{2}$ boundary whose inward mean curvature satisfies $\mathcal{H}>0$. Then the Dirichlet problem

$$
\begin{aligned}
u_{t} & =g^{i j} D_{i} D_{j} u \text { in } \Omega \times(0, \infty) \\
u(x, t) & =\phi(x) \text { on } \partial \Omega \times(0, \infty) \\
u(x, 0) & =u_{0}(x)
\end{aligned}
$$

is solvable for arbitrary continuous boundary data $\phi$ and $C^{2}(\Omega) \cup C^{0}(\bar{\Omega})$ compatible initial data $u_{0}$. Moreover as $T \rightarrow \infty, u(x, t)$ converges to the solution of the minimal surface equation with boundary data $\phi(x)$ given by Theorem 1.4.

Example 1.8. It is probably useful at this point to give an example which shows that there cannot be an interior gradient estimate of the form $W(P) \leq f\left(\frac{u(P)}{\rho}\right)$ Let $M$ be $\mathbf{H}^{2}$, two dimensional hyperbolic space and take $H(x) \equiv \frac{1}{2}$. At a fixed point, we choose geodesic polar coordinates $d s^{2}=d r^{2}+\sinh ^{2} r d \theta^{2}$. We look for a solution of (1.1) of the form $u=u(r)$ giving the ode

$$
\frac{1}{\sinh r}\left(\sinh r \frac{u^{\prime}}{\sqrt{1+u^{\prime 2}}}\right)^{\prime}=1 \text {. }
$$


Integrating once gives

$$
\frac{u^{\prime}}{\sqrt{1+u^{\prime 2}}}=\frac{\cosh r-1}{\sinh r}
$$

which yields $u^{\prime}=\sqrt{\frac{\cosh r-1}{2}}$ and so $u=\sqrt{2(\cosh r+1)}$. Thus we have positive entire graphs of constant mean curvature, unlike the situation for Euclidean space.

For non-compact $M^{n}$, the existence of entire constant mean curvature graphs is closely related to Cheeger's isoperimetric constant

$$
h(M)=\inf _{\Omega} \frac{|\partial \Omega|}{|\Omega|},
$$

where $\Omega$ ranges over all compact domains in $\mathrm{M}$ with rectifiable boundaries. Using the divergence form of (1.1), we see that if there is an entire $\mathrm{H}$ graph , then necessarily $H \leq \frac{|\partial \Omega|}{|\Omega|}$ for all bounded $\Omega$ and so $H \leq h(M)$. For example in $H^{n}$, this gives the condition $H \leq \frac{n-1}{n}$ which is sharp. Note also that in this case, the condition $\operatorname{Ric}(M) \geq-\frac{n^{2}}{n-1} H^{2}$ is exactly $H \geq \frac{n-1}{n}$. When the opposite inequality is satisfied, there is a non-negative entire solution, which implies apriori height estimates for the Dirichlet problem. For recent work on sharp height estimates in $M^{2} \times R$, see [17].

In future work, we will study other curvature functions such as the "higher order mean curvatures" $H_{r}(\kappa)=\frac{\sigma_{r}(\kappa)}{\sigma_{r}(\overrightarrow{1})}$. We just want to point out that the Dirichlet problem for extrinsic Gauss curvature $K_{0}>0$ is precisely

$$
\begin{aligned}
\frac{\operatorname{det} D_{i} D_{j} u}{\sigma} & =K_{0} W^{n+2} \text { in } \Omega \\
u & =\phi \text { on } \partial \Omega .
\end{aligned}
$$

From the work of Bo Guan [4], we have

Theorem 1.9.. Suppose there exists a locally strictly convex subsolution $\underline{u}$, that is,

$$
\begin{aligned}
\frac{\operatorname{det} D_{i} D_{j} u}{\sigma} & \geq K_{0} W^{n+2} \text { in } \Omega \\
u & =\phi \text { on } \partial \Omega .
\end{aligned}
$$


Then there exists a stricly convex solution $u \in C^{\infty}(\bar{\Omega})$ of the Dirichlet problem (1.19).

\section{Local CALCUlations FOR THE GRAPH S}

Let $x_{1}, \ldots x_{n}$ be a system of local coordinates for $\mathrm{M}$ with corresponding metric $\sigma_{i j}$. Then the coordinate vector fields for $\mathrm{S}$ and the upward unit normal to $\mathrm{S}$ is given by

$$
X_{i}=\frac{\partial}{\partial x_{i}}+u_{i} \frac{\partial}{\partial t}
$$

and

$$
N=\frac{1}{W}\left(-u^{j} \frac{\partial}{\partial x_{j}}+\frac{\partial}{\partial t}\right), u^{i}=\sigma^{i j} u_{j}
$$

The induced metric on $\mathrm{S}$ is then

$$
g_{i j}=<X_{i}, X_{j}>=\sigma_{i j}+u_{i} u_{j}
$$

with inverse

$$
g^{i j}=\sigma^{i j}-\frac{u^{i} u^{j}}{W^{2}}
$$

We claim that

$$
g=\operatorname{det}\left(g_{i j}\right)=\sigma W^{2}, \sigma=\operatorname{det}\left(\sigma_{i j}\right) .
$$

To see this, assume $|D u| \neq 0$ and note that

$$
\left[\left(\sigma^{i j}\right)\left(g_{i j}\right)\right]_{k l}=\sigma^{k i}\left(\sigma_{i l}+u_{i} u_{l}\right)=\delta_{k l}+u^{k} u_{l} .
$$

Hence $\sigma^{-1} g=\operatorname{det}\left(\delta_{k l}+u^{k} u_{l}\right)=W^{2}$ since $\left(\delta_{k l}+u^{k} u_{l}\right)$ has eigenvalues 1 with multiplicity $n-1$ (eigenvectors orthogonal to the Euclidean gradient of $\mathrm{u}$ ) and eigenvalue $W^{2}$ with eigenvector parallel to the Euclidean gradient of $\mathrm{u}$.

The second fundamental form $b_{i j}$ of $\mathrm{S}$ is given by $(\bar{D}$ is covariant differentiation on $M \times R)$

$$
\begin{gathered}
b_{i j}=<\bar{D}_{X_{i}} X_{j}, N>=<D_{\frac{\partial}{\partial x_{i}}} \frac{\partial}{\partial x_{j}}+u_{i j} \frac{\partial}{\partial t}, N> \\
=<\Gamma_{i j}^{k} \frac{\partial}{\partial x_{k}}+u_{i j} \frac{\partial}{\partial t}, N>=\frac{1}{W}\left(-\Gamma_{i j}^{k} u^{l} \sigma_{k l}+u_{i j}\right) .
\end{gathered}
$$


Hence,

$$
b_{i j}=\frac{D_{i} D_{j} u}{W}
$$

and so the equation of prescribed mean curvature $H(x)$ is then

$$
n H(x)=\frac{1}{W} g^{i j} D_{i} D_{j} u .
$$

Now consider the functional $I(S)$ representing the area of S plus the weighted volume under the graph. In local coordinates,

$$
I(S)=\int(W+n H(x) u(x)) \sqrt{\sigma} d x .
$$

As a functional of $\mathrm{u}$, this gives the equation of prescribed mean curvature $H(x)$ as Euler-Lagrange equation

$$
\operatorname{div} \frac{D u}{W}=\frac{1}{\sqrt{\sigma}} D_{i}\left(\frac{u^{i}}{W}\right)=n H(x) .
$$

It is easily seen that (2.25) is the non-divergence form of (2.26).

We will also need the well known formulae

$$
\begin{aligned}
\Delta_{S} u & =\frac{n H(x)}{W} \\
\Delta_{S} \frac{1}{W} & =-\left(|A|^{2}+\operatorname{Ric}(N)+N(H(x))\right) \frac{1}{W},
\end{aligned}
$$

where $|A|$ is the norm of the second fundamental form, Ric is the Ricci curvature of $M \times R, N(H)$ is the directional derivative of $\mathrm{H}(\mathrm{x})$ in the normal direction $\mathrm{N}$ and $\Delta_{S}$ is the Laplace-Beltrami operator of $\mathrm{S}$ given in local coordinates by

$$
\Delta_{S} \equiv \operatorname{div}_{S}\left(D_{S} \cdot\right)=\frac{1}{\sqrt{g}} D_{i}\left(\sqrt{g} g^{i j} D_{j} \cdot\right) .
$$

For a clean derivation of (2.28) using moving frames see [13, section 2] where $\mathrm{M}$ is three dimensional but the derivation is valid in all dimensions. Equation (2.28) is easily seen to be equivalent to (2.26).

For a discussion of $\Delta_{S}$ in terms of $\operatorname{div}_{S}$ see [6], where the important formula

$$
\Delta_{S} \phi(x)=g^{i j} D_{i} D_{j} \phi-n H(x) \frac{u^{k}}{W} \phi_{k} .
$$


is derived. From (2.30) follows the useful formula

$$
\Delta_{S} g(\phi)=g^{\prime}(\phi) \Delta_{S} \phi+g^{\prime \prime}(\phi) g^{i j} D_{i} \phi D_{j} \phi .
$$

\section{The Proof of Theorems 1.1 And 1.3}

We will derive a maximum principle for the function $h=\eta(x) W$ by computing $\Delta_{S} h$. From (2.28) and (2.31) we find

$$
\Delta_{S} W-\frac{2}{W} g^{i j} D_{i} W D_{j} W \geq-C W .
$$

Then a simple computation gives

$$
\begin{aligned}
L h & \equiv \Delta_{S} h-2 g^{i j} \frac{D_{i} W}{W} D_{j} h \\
& =\eta\left(\Delta_{S} W-\frac{2}{W} g^{i j} D_{i} W D_{j} W\right)+W \Delta_{S} \eta \geq W\left(\Delta_{S} \eta-C \eta\right) .
\end{aligned}
$$

We define

$$
\eta(x) \equiv g(\phi(x)) ; \quad g(\phi)=e^{K \phi}-1,
$$

with the constant $K>0$ to be determined and

$$
\phi(x)=\left(-\frac{u(x)}{2 u_{0}}+\left(1-\left(\frac{d(x)}{\rho}\right)^{2}\right)\right)^{+} .
$$

Here $d(x)$ is the distance function from $\mathrm{P}$, the center of the geodesic ball $B_{\rho}(P) \subset \Omega$ and we will bound $W(P)$. Since $\mathrm{u}$ is non-negative, $\eta(x)$ vanishes outside of $\partial B_{\rho}(P)$. To be more precise, we should replace 1 by 1- $\epsilon$ in the definition of $\phi$ so that $\eta$ is smooth with compact support and later let $\epsilon$ tend to zero. For simplicity of writing, we will omit this.

The point is now to choose $\mathrm{K}$ so that $\Delta_{S} \eta-C \eta>0$ on the set where $h>0$ and $\mathrm{W}$ is large. A straightforward compution gives that on the set where $h>0$,

$$
\Delta_{S} \eta-C \eta \geq W e^{K \phi}\left\{K^{2}\left(\frac{|D u|^{2}}{4 u(P)^{2} W^{2}}-2 \frac{|D u|}{\rho^{2} W^{2}}\right)-C K\left(\frac{1}{u(P)}+\frac{1}{\rho^{2}}\right)-C\right\}
$$

Hence on the set where $h>0$ and $W>16 \max \left(1,\left(\frac{u(P)}{\rho}\right)^{2}\right)$, we find

$$
\Delta_{S} \eta-C \eta \geq W e^{K \phi}\left\{\frac{K^{2}}{8 u(P)^{2}}-C K\left(\frac{1}{u(P)}+\frac{1}{\rho^{2}}\right)-C\right\}
$$


We now choose $K=M u(P)\left(1+\frac{u(P)}{\rho^{2}}\right)$ where $\mathrm{M}$ is large but independent of $u(P)$ and $\rho$. Then

$$
\Delta_{S} \eta-C \eta \geq W e^{K \phi}\left\{\left(\frac{M^{2}}{8}-C M\right)\left(1+\frac{u(P)}{\rho^{2}}\right)-C\right\}>0,
$$

for $M=16 C$. Hence by (3.34) and the maximum principle, $W \leq 16 \max \left(1,\left(\frac{u(P)}{\rho}\right)^{2}\right)$ at the point where $h$ achieves its maximum. Therefore

$$
h(P)=\left(e^{\frac{K}{2}}-1\right) W(P) \leq\left(e^{K}-1\right) \cdot 16 \max \left(1,\left(\frac{u(P)}{\rho}\right)^{2}\right)
$$

or

$$
W(P) \leq 32 \max \left(1,\left(\frac{u(P)}{\rho}\right)^{2}\right) e^{16 C u(P)} e^{16 C\left(\frac{u(P)}{\rho}\right)^{2}},
$$

which proves Theorem 1.1.

The proof of Theorem 1.3 is very similar and follows the argument of EvansSpruck [1].

For later use in the proof of Theorem 1.4, it is convenient to have a global maximum principle for $W$ assuming that $\mathrm{u}$ is globally bounded.

Theorem 3.1.. Let $u \in C^{3}(\Omega)$ be a solution of (1.1) where $H(x) \geq H>0$ and assume $|u| \leq M$. Then

$$
\sup _{\Omega} W \leq e^{2 C M} \sup _{\partial \Omega} W+2 .
$$

Proof. From (3.34) with $\eta=e^{K u}$ we find

$$
L h=K h\left(\frac{n H(x)}{W}-\frac{K}{W^{2}}+\left(K-\frac{C}{K}\right)\right) \geq K h\left(\frac{n H}{W}-\frac{K}{W^{2}}+\left(K-\frac{C}{K}\right)\right) .
$$

We choose $K=C$ and note that at an interior maximum of $\mathrm{h}$,

$$
(C-1) W^{2}+n H W \leq C,
$$

which implies $W \leq 2$. On the other hand if $\mathrm{h}$ assumes its maximum on $\partial \Omega$,

$$
\sup _{\Omega} W \leq e^{2 C M} \sup _{\partial \Omega} W .
$$

Combining the two cases proves Theorem 3.1. 


\section{The Proof of Theorems 1.4 And 1.5}

The main step is to obtain a global apriori estimate for $\sup _{\Omega}|u|$ and $\sup _{\partial \Omega}|\nabla u|$ for a family of solutions $u^{t}$ of the constant mean curvature equation with mean curvature $t H$ and boundary values $t \phi 0 \leq t \leq 1$, independent of $t$. For Theorem 1.5, in step 1, we use Theorem 1.2 which we prove in the next section.

Since the argument is the same for all $t$, we will do this for $t=1$. Then by Theorem 3.1 (global gradient bound), we have a uniform bound for the $\left|u^{t}\right|_{C^{1}(\Omega)}$ and the remainder of the argument is standard.

Step 1. Estimation of $\sup _{\Omega}|u|$

Proposition 4.1.. (yy li and nirenberg) Assume $\partial \Omega \in C^{2}$ and let $\Omega^{0}$ be the largest open subset of points $x \in \Omega$ which have a unique closest point $y \in \partial \Omega$. The the distance function $d(x)$ to $\partial \Omega$ is $C^{2}\left(\Omega^{0}\right)$.

Lemma 4.2.. Assume Ric $\geq-\frac{n^{2}}{n-1} H^{2}$ and $\mathcal{H}_{\partial \Omega} \geq \frac{n}{n-1} H$. For $x_{0} \in \Omega^{0}$, let $\mathcal{H}\left(x_{0}\right)$ be the (inward) mean curvature of the level set of $d(x)$ passing though $x_{0}$. Then $\mathcal{H}\left(x_{0}\right) \geq \frac{n}{n-1} H$.

Proof. Let $H(t)$ be the mean curvature of the level set at a point where $d(x)=t$ along the geodesic $\sigma(t)$ joining $y_{0} \in \partial \Omega$ to $x_{0}$. By standard comparison theory

$$
H^{\prime}(t) \geq H^{2}(t)+\frac{1}{n-1} \operatorname{Ric}\left(\sigma^{\prime}(t), \sigma^{\prime}(t)\right) \geq H^{2}(t)-\left(\frac{n}{n-1} H\right)^{2} .
$$

Since $H(0)=\mathcal{H}_{\partial \Omega} \geq \frac{n}{n-1} H$, it follows that $H^{\prime}(t) \geq 0$ and the lemma follows.

We now construct an upper barrier for - $\mathrm{u}$ (i.e a lower barrier for $\mathrm{u}$ ) of the form

$$
w=\sup -\phi+h(d(x))
$$


where $\mathrm{d}(\mathrm{x})$ is the distance function to $\partial \Omega$. Then if $x \in \Omega^{0}$,

$$
\begin{aligned}
M w & =\frac{1}{W}\left(\sigma^{i j}-\frac{w^{i} w^{j}}{W^{2}}\right)\left(h^{\prime} D_{i} D_{j} d+h^{\prime \prime} D_{i} d D_{j} d\right) \\
& =\frac{1}{\sqrt{1+h^{\prime 2}}}\left(h^{\prime} \Delta d+\frac{h^{\prime \prime}}{\left(1+h^{\prime 2}\right)}\right) \\
& =\frac{h^{\prime \prime}}{\left(1+h^{\prime 2}\right)^{\frac{3}{2}}}-\frac{h^{\prime}}{\sqrt{1+h^{\prime 2}}}(n-1) \mathcal{H}(x) \\
& \leq \frac{h^{\prime \prime}}{\left(1+h^{\prime 2}\right)^{\frac{3}{2}}}-n H \frac{h^{\prime}}{\sqrt{1+h^{\prime 2}}} .
\end{aligned}
$$

Choose $h=\frac{e^{A C}}{C}\left(1-e^{-C d}\right)$ where $A>2 \max _{\Omega} d$ is fixed and $\mathrm{C}$ is large. Then $h^{\prime}=e^{C(A-d)}$ and $h^{\prime \prime}=-C h$ and so

for $C>n H$.

$$
M w \leq-\frac{C h^{\prime}}{\left(1+h^{\prime 2}\right)^{\frac{3}{2}}}-n H \frac{h^{\prime}}{\sqrt{1+h^{\prime 2}}} \leq-n H
$$

Let $v=-u$. We Claim: $v \leq w$ (we show that $\mathrm{w}$ is a viscosity supersolution)

Suppose $M=\sup v-w>0$ is achieved at $x_{0}$ and let $y_{0} \in \partial \Omega$ be the closest point to $x_{0}$. Then since $v(x)-v\left(x_{0}\right) \leq w(x)-w\left(x_{0}\right)$, a simple argument show that $\mid \nabla v\left(x_{0} \mid\right)=h^{\prime}\left(d\left(x_{0}\right)>0\right.$ so the local level set

$$
\Gamma=\left\{x \in \Omega: v(x)=w\left(x_{0}\right)+M\right\}
$$

is $C^{2}$ near $x_{0}$ and $w(x) \geq w\left(x_{0}\right)$ on $\Gamma$. Since $\mathrm{h}$ is increasing this means that $d(x) \geq d\left(x_{0}\right)$ on $\Gamma$.

Hence we can find a small ball $B_{\epsilon}\left(z_{0}\right)$ tangent to $\Gamma$ at $x_{0}$ such that

$$
w(x)+M \geq v(x) \geq w\left(x_{0}\right)+M \text { on } B_{\epsilon}\left(z_{0}\right) .
$$

So $w(x) \geq w\left(x_{0}\right)$, i.e. $d(x) \geq d\left(x_{0}\right)$ on $B_{\epsilon}\left(z_{0}\right)$

Therefore the ball of radius $d\left(x_{0}\right)+\epsilon$ centered at $z_{0}$ is contained in $\bar{\Omega}$ and thus $z_{0}$ is on the extension of the geodesic from $y_{0}$ to $x_{0}$ (otherwise there exists $z \in B_{\epsilon}\left(z_{0}\right)$ with $d(z)<d\left(x_{0}\right)$, a contadiction). 
Hence $x_{0} \in \Omega^{0}$ and our earlier computation shows this violates the maximum principle. This completes Step 1.

Step 2. Estimation of $\sup _{\partial \Omega}|\nabla u|$

This argument is now standard and uses a barrier $w=\phi(x)+h(d)$ where $h(0)=0$ in a neighborhood $\left\{0<d(x)<d_{0}\right\}$ of $\partial \Omega$. For details see [3].

Step 3. Existence for continuous $\phi(x)$.

When $\phi(x)$ is only continuous we approximate $\phi$ uniformly by smooth boundary data and use the interior gradient estimate to obtain strong convergence on compact subsets of $\Omega$.

A local barrier argument show that the limiting solution achieves the boundary values $\phi$

\section{OTHER WAYS TO OBTAIN SHARP SUP NORM ESTIMATES}

5.1. Moser iteration when the sharp isoperimetric (Sobolev) inequality holds $n=2,3,4$ and $K_{\pi} \leq 0$. When the sharp Euclidean Sobolev inequality holds, then the assumption $\int_{\Omega}|H(x)|^{n} d V<\omega_{n}$ implies a sup norm estimate (see [3] p. 276).

For $n=2$ and $K_{\pi} \leq-1$ say, the sharp isoperimetric inequality

$$
L^{2} \geq 4 \pi A+A^{2}
$$

leads to the sharp Sobolev inequality

$$
\begin{aligned}
\left(\int_{\Omega}|\nabla v| d V\right)^{2} & \geq 4 \pi \int_{\Omega} v^{2} d V+\left(\int_{\Omega}|v| d V\right)^{2} \\
& \geq\left(\frac{4 \pi}{\Omega}+1\right)\left(\int_{\Omega}|v| d V\right)^{2}
\end{aligned}
$$

$\forall v \in H_{0}^{1}(\Omega)$. Using this improved Sobolev inequality as in [3, Theorem 10.10] gives 
Proposition 5.1.. Let $M^{2}$ be complete, simply connected with sectional curvatures $K_{\pi} \leq-1$ Let $u$ satisfy div $\frac{D u}{W}=n H$ in $\Omega \subset M$. Then if $4 H^{2}<1+\frac{4 \pi}{\Omega}$,

$$
\sup _{\Omega}|u| \leq \sup _{\partial \Omega}|u|+C
$$

for a uniform constant $C$. In particular, the last condition is always satisfied for $0 \leq H \leq \frac{1}{2}$

Example 5.2.. In $H^{2}$ let $\Omega=B_{R}$. Then $|\Omega|=2 \pi(\cosh R-1)$ and for

$$
\cosh R=\frac{4 H^{2}+1}{4 H^{2}-1}, H>\frac{1}{2}
$$

we have the equality

$$
4 H^{2}-1=\frac{4 \pi}{\Omega}
$$

and we have a radial "hemisphere" solution characterized by the ode

$$
\frac{u^{\prime}(r)}{\sqrt{1+u^{\prime 2}}}=2 H \frac{\cosh r-1}{\sinh r} .
$$

Theorem 5.3..(after Bernstein-Finn) If $u$ is any solution in $B_{R}$ then $u$ is the hemisphere solution (modulo a vertical translation).

The proof is the same as in Finn's paper [2] using the hemisphere solution as a barrier..

5.2. Entire solutions in $H^{n}$. For $H^{n}$, Ric $=-(n-1) \sigma$ and so the Ricci curvature condition in Theorem 3.1, is precisely satisfied when $H \geq \frac{n-1}{n}$. For $0 \leq H \leq \frac{n-1}{n}$, analogous to the $n=2$ case, there is a complete entire positive radial solution. Such a solution always give sup norm estimates by the maximum principle.

Theorem 5.4.. Let $\Omega \subset H^{n}$ satisfy $\mathcal{H}_{\partial \Omega} \geq \frac{n}{n-1} H \quad$ (with strict inequality for $0<H<\frac{n-1}{n}$ ) for a non-negative constant $H$. Then the Dirichlet problem for constant mean curvature graphs has a solution for arbitrary continuous boundary values $\phi(x)$. 


\section{The Proof of Theorem 1.6.}

We sketch the proof of Theorem 1.6 since it is basically well known. From (2.28) we obtain (using that $|A|^{2} \geq n H^{2}$ ) that $\frac{1}{W}$ and $H u+\frac{1}{W}$ are superharmonic on $\mathrm{S}$, the graph of $u$. In particular $0 \geq u \geq-\frac{1}{H}$ and both functions achieve their minimum on $\partial \Omega$ at the same point $\mathrm{P}$ where $|\nabla u|$ achieves its maximum.

Introduce a local orthonormal frame $e_{1}, \ldots, e_{n}$ at $\mathrm{P}$ with $e_{n}$ the interior unit normal direction and $e_{\alpha}, \alpha<n$ the principal curvature directions to $\partial \Omega$ at $\mathrm{P}$. Then $\nabla_{n}\left(H u+\frac{1}{W}\right)=H u_{n}-\frac{u_{n}}{W^{3}} \nabla_{n n} u \leq 0$ at P, i.e. ,

$$
\nabla_{n n} u \geq H W^{3} \text { at } \mathrm{P},
$$

and also

$$
\sum_{\alpha<n} \nabla_{\alpha \alpha} u=(n-1)\left|u_{n}\right| \mathcal{H} \geq(1+\epsilon)(n-1) H\left|u_{n}\right| .
$$

On the other hand, using the nondivergence form (1.2) of the equation gives at $\mathrm{P}$,

$$
n H W=\sum_{\alpha<n} \nabla_{\alpha \alpha} u+\frac{\nabla_{n n} u}{W^{2}}
$$

Combining (6.42),(6.43)(6.44) yields

$$
\frac{\left|u_{n}\right|}{W} \leq \frac{1}{1+\epsilon}
$$

that is $|\nabla u|(P) \leq \frac{1}{\sqrt{\epsilon^{2}+2 \epsilon}}$.

Now that we have $C^{1}$ estimates, the theorem follows as before.

\section{The PRoof of Theorem 1.2.}

We sketch the proof of Theorem 1.2 . The first step is to show that solutions are always uniformly bounded below in a fixed neighborhood of the boundary.

Lemma 7.1.. Let $u$ be as in Theorem 1.2. Then $u>-\log 2$ in a fixed neighborhood $\Omega_{\sigma}=\left\{x \in \Omega: 0<d(x)<\frac{1}{\sigma}\right\}$ for fixed $\sigma>>\frac{1}{\epsilon}$. 
Proof. Consider the barrier $w=\log \left(1-\frac{\sigma}{2} d(x)\right)$. Then as in Step $1, M w \geq$ $n H$ in $\Omega_{\frac{1}{2} \sigma}$. Moreover, $w=0$ on $\partial \Omega$ and $w=-\infty$ on $d(x)=\frac{2}{\sigma}$. Thus $u \geq$ $w$ on $\partial \Omega_{\frac{1}{2} \sigma}$ and so $u \geq w$ in $\Omega_{\frac{1}{2} \sigma}$ by the maximum principle for the difference of solutions. In particular, $u>-\log 2$ in $\Omega_{\sigma}$.

Suppose now that Theorem 1.2 is false. Then there is a sequence of solutions $u_{n}$ in $\Omega$ with $u_{n} \geq 0$ on $\partial \Omega$ and $\inf u_{n}=u_{n}\left(P_{n}\right)=-n$. Note that by Lemma 7.1, $d\left(P_{n}\right)>\frac{1}{\sigma}$ and so we may assume by choosing a subsequence that $P_{n} \rightarrow P \in \Omega$. Now let $v_{n}=u_{n}+n \geq 0$ and note that $v_{n} \geq n-\log 2$ in $\Omega_{\sigma}$ and $v_{n}\left(P_{n}\right)=0$. We now use Theorem 1.1 as in Serrin [15] to prove a local Harnack inequality for $v_{n}$. That is , the sequence $v_{n}$ remains bounded in a small fixed neighborhood of $\mathrm{P}$ and thus (by Schauder estimates) converges uniformly to a solution $\mathrm{v}$ in a maximal open neighborhood $\mathrm{D}$ of $\mathrm{P}$ and diverges to $+\infty$ on $\Omega \backslash D$. But this situation is impossible for we can translate $\mathrm{v}$ up until it is above $v_{1}$ in $\mathrm{D}$ and then lower it until there is a first contact. This first contact must be over an interior point of $\mathrm{D}$, violating the maximum principle.

\section{REFERENCES}

[1] Evans, L. C. and Spruck, J., Motion of level sets by mean curvature III, J. Geom. Anal. 5(1992), 121-150.

[2] Finn, R., Remarks relevant to minimal surfaces and to surfaces of prescribed mean curvature, J. D'Analyse Math. 14(1965), 139-160.

[3] Gilbarg, D. and Trudinger, N., Elliptic Partial Differential Equations of Second Order, 2nd ed., Springer-Verlag, New York-Heidelberg-Berlin, 1983.

[4] Guan, Bo, The Dirichlet problem for Monge-Ampere equations in non-convex domains and spacelike hypersurfaces of constant Gauss curvature, Trans. AMS 350(1998), 4955-4971.

[5] Guan, Bo and Spruck, J., Interior gradient estimates for solutions of prescribed curvature equations of parabolic type, Indiana Univ. Math. J . 40(1991), 1471-1481.

[6] Hoffman, D. and Spruck, J., Sobolev and Isoperimetric inequalities for Riemannian submanifolds, Comm. Pure and Appl. Math 27(1974), 715-727.

[7] Huisken, G., Nonparametric mean curvature flow with boundary conditions, J. Diff. Geom. 77(1989), 369-378.

[8] Korevaar, N.J., A priori interior gradient bounds for solutions to elliptic Weingarten equations, Ann. Inst. Henri Poincare 4(1987), 405-421.

[9] Li, Y.Y and Nirenberg, L., Regularity of the distance function to the boundary, preprint.

[10] Li, Y.Y and Nirenberg, L., The distance function to the boundary, Finsler Geometry and the singular set of viscosity solutions of some Hamilton-Jacobi equations, Comm. Pure Appl. Math 58 (2005), 85-145. 
[11] Oliker, V. I. and Uraltseva, N. N., Long time behavior of flows moving by mean curvature II, Topol. Methods Nonlinear Anal. 9 (1997), 17-28.

[12] Oliker, V. I. and Uraltseva, N. N., Evolution of nonparametric surfaces with speed depending on curvature II, Comm. Pure Applied Math. 46 (1993), 97-135.

[13] Schoen, R. and Yau, S. T., Proof of the positive mass theorem II, Comm. Math. Phys. 79(1981), 231-260.

[14] Serrin, J., The problem of Dirichlet for quasilinear elliptic differential equations with many independent variables, Philos. Trans. Roy. Soc. London Ser. A 264(1969), 413-496.

[15] Serrin, J., The Dirichlet problem for surfaces of constant mean curvature, Proc. London Math. Soc. 21(1970), 361-384.

[16] Spruck, J., Infinite boundary value problems for surfaces of constant mean curvature, Arch. Rat. Mech. Anal. 49(1972), 1-31.

[17] Aledo, J. A., Espinar, J. H. and Gálvez, J.A., Height estimates for surfaces with positive constant mean curvature in $M^{2} \times R$, to appear in Illinois J. Math.

\section{Joel Spruck}

Department of Mathematics

Johns Hopkins University Baltimore, MD 21218.

E-mail: js@math.jhu.edu 